\title{
Horizons/Théâtre
}

Revue d'études théâtrales

\section{Rejouer la ville, rejouer le réel : Grand Magasin ou les enjeux de la prévision}

\section{Élisabeth Magne}

\section{(2) OpenEdition}

1 Journals

Édition électronique

URL : https://journals.openedition.org/ht/795

DOI : $10.4000 /$ ht.795

ISSN : 2678-5420

Éditeur

Presses universitaires de Bordeaux

\section{Édition imprimée}

Date de publication : 31 décembre 2016

Pagination : 84-96

ISSN : 2261-4591

\section{Référence électronique}

Élisabeth Magne, «Rejouer la ville, rejouer le réel : Grand Magasin ou les enjeux de la prévision »,

Horizons/Théâtre [En ligne], 8-9 | 2016, mis en ligne le 01 janvier 2018, consulté le 25 mai 2022. URL : http://journals.openedition.org/ht/795 ; DOl : https://doi.org/10.4000/ht.795

\section{(c) (i) $\odot$}

La revue Horizons/Théâtre est mise à disposition selon les termes de la Licence Creative Commons Attribution - Pas d'Utilisation Commerciale - Pas de Modification 4.0 International. 


\section{ÉLISABETH MAGNE}

Élisabeth Magne est maître de conférences en arts plastiques à l'université Bordeaux Montaigne depuis 2006. Ses recherches portent sur les processus d'instauration des œuvres d'art et notamment des œuvres contemporaines (matériaux, outils, protocoles etc.). Elle travaille également sur les usages et pratiques anthropologiques des images. Elle appartient au laboratoire CLARE/Artes.

Mail : Elisabeth.Magne@u-bordeaux-montaigne.fr

Résumé : Trois exemples de pratique théâtrale, plasticienne et vidéographique sont ici étudiés pour leurs qualités de reprise fictionnelle d'un réel dont ils s'écartent très peu au point de brouiller les frontières entre expérience première et rejeu : Prévisions, événements scénarisés par le collectif Grand Magasin, le catalogue de La Déroute produit par Nicolas Simarik et La Bataille d'Orgreave reconstituée et filmée par Jeremy Deller. Regardés sous l'angle de la sociologie d'Ervin Goffman, ces objets produisent tous trois de l'incertitude et du questionne-

Abstract: Three examples of theater art, visual art, and video art are here studied for their qualities of fictional recovery of a "real life" from which they deviate very little, to the point of blurring the frontiers between first-hand experience and replay: Prévisions/Forecast, events scripted by the collective Grand Magasin, the catalogue of La Déroute/The Rout produced by Nicolas Simarik, and La Bataille d'Orgreave/The Battle of Orgreave reconstructed and filmed by Jeremy Deller. Observed from the sociological perspective of Ervin Goffman, all of ment critique quant aux cadres dans lesquels s'inscrivent nos interactions quotidiennes. Jover, ici, c'est ouvrir la pensée sur le réel si proche, c'est donner à voir des expériences paradigmatiques qui éclairent le continuum indifférencié, la confusion ordinaire.

Mots-cLés : jouer, rejouer, jeu, réel, théâtralité, reconstitution historique, art contextuel, cadre de l'expérience

these three objects produce uncertainty and critical questioning concerning the frameworks in which our daily interactions are inscribed. Here, to play is to open thinking of the real so close, it's to make visible the paradigmatic experiences that illuminate the undifferentiated continuum, the ordinary confusion.

KEYwords: replay, role playing, game, reality, reenactment, contextual art, framework 


\section{Rejouer la ville, rejouer le réel : Grand Magasin ou les enjeux de la prévision}

En 2011, la commune de Vernier près de Genève lance sa première Biennale d'Art contemporain et invite le collectif Grand Magasin à s'installer pour une création dans le quartier des Libellules. Pascale Murtin et François Hiffler ont déjà une solide expérience des pratiques interventionnistes en tout genre : spectacles, pseudo-conférences, événements, happenings sans cadre, ces deux-là se plaisent à brouiller les frontières du réel et de la mise en fiction des territoires où ils travaillent. Sur place, il y a quelques barres d'immeubles en habitat collectif comme on en trouve un peu partout et des gens qui vont et viennent selon les aléas d'un quotidien plus ou moins prévisible.

À Vernier, du 22 au 25 septembre 2011, Grand Magasin crée Prévisions, « série d'événements discrets se succédant irrégulièrement $»^{1}$ dans et autour de ces immeubles pendant les quatre jours que dure la manifestation : «Un livret, disponible sur place, décrit par avance chaque action, en précise l'heure exacte et l'emplacement. Il s'agit d'actions peu spectaculaires, à peine remarquables s'apparentant à l'activité quotidienne, ponctuellement effectuées par de nombreux complices (monter ou descendre un escalier, emprunter un ascenseur, laver ou garer sa voiture). Les faits annoncés se produisent comme par miracle en temps et lieu, qu'il y ait des spectateurs ou pas $\gg^{2}$. Prévisions, c'est une série d'annonces, comme la météo, mais avec un taux de fiabilité plus important : « Le jeu peut consister, pour le visiteur curieux, à vérifier que le fait prédit a effectivement lieu ou, pour les plus paresseux, à simplement lire les pronostics $\gg^{3}$. Le programme change sans cesse et tourne de $15 \mathrm{~h}$ à $20 \mathrm{~h}$ tous les jours avec une précision digne des fiches horaires de la SNCF. En voici quelques exemples prélevés dans le livret :

vendredi 23 septembre 2011

$17 h 45$

Une femme et un homme

sur les rochers du Parking de l'école. 
Lui : barbe, lunettes, chapeau.

Ils surveillent d'un cil leur jeune fils.

$17 h 50$

Un cycliste quittant la route de l'Usine-à-Gaz

remonte l'allée qui surplombe le stade

en actionnant plusieurs fois sa sonnette.

samedi 24 septembre 2011

$17 \mathrm{~h} 14$

Sur l'allée surplombant le Stade

une fermme est debout entre deux bancs.

Elle porte une veste verte.

Un avion passe,

puis un second.

dimanche 25 septembre 2011

$14 h 40$

Un cycliste au coupe-vent orange

passe avenue des Libellules.

Il marque une pause

devant le panneau horaire de l'autobus 51 .

$15 h 07$

Devant la porte 8,

un homme sort des bouteilles d'eau

du coffre de la Clio 6528 YE $22 .^{4}$

La littéralité descriptive de ces petits textes est assez savoureuse et attribue un rôle aussi bien à l'avion de $17 \mathrm{~h} 14^{5}$ qu'à quelques habitants. On comprend aisément comment l'affaire s'est écrite et quel fut le point de départ : une observation patiente du réel, un regard attentif sur des micro-événements, notés, archivés, isolés comme des items singuliers dans la liste sans fin de l'affairement de la fourmilière, puis inscrits dans le protocole pour une réitération planifiée, ou inventés de toutes pièces pour les besoins de l'affaire. Peu importe finalement. Seule compte la nature de ces actions : des événements minuscules dont aucun ne fait vraiment saillie avec le continuum du temps 
ordinaire, dépouillés des marques de la fiction, dilués dans les activités quotidiennes d'une communauté qui vaque à ses occupations.

Le déroulé s'inscrit dans une chronologie largement incomplète, faite de plus de vides que de pleins. Si l'on n'a pas le livret sous les yeux, vont se produire des choses dont on ne saura pas si elles appartiennent au champ de l'intervention, jusqu'à confondre sans doute ce qui est prévu et ce qui ne l'est pas. La méprise est évidemment bienvenue et révèle sans doute la scénarisation involontaire d'un réel parfois prévisible à l'insu de toute présence spectatrice.

À ce jeu du brouillage des frontières du champ et du hors champ, Grand Magasin n'a rien inventé. D'autres compagnies de théâtre s'y sont frottées comme Royal de Luxe dans un spectacle intitulé Roman photo : tournage créé en 1987, un pseudo-tournage de roman photo installé dans le flot des passagers d'une gare aux heures de pointe. Si la mise en fiction s'imposait assez vite comme une évidence, l'arrivée des comédiens dans les mouvements des passagers de la gare laissait libre cours à l'imagination. Tel hurluberlu fonçant depuis son quai se révélait être un quidam ordinaire, tout comme deux curés en soutane ou la starlette encore éméchée au petit matin. Impossible de distinguer qui venait pour jouer et qui passait là par hasard, l'incertitude étant totale jusqu'au dernier moment. Avec en filigrane une pensée fugitive : le monde ordinaire ne l'est finalement pas tant que cela et la foule est un spectacle en soi, brouillant volontairement les repères et le cadrage d'une telle prestation.

Avec Prévisions, la matière fictionnelle est tellement « raccord $\gg$ avec le réel qu'elle en devient invisible. Le cadre se fait transparent jusqu'à disparaitre ${ }^{6}$.

Seul le livret fabrique la représentation et prévoit ce qui doit se passer selon un calendrier rigoureux : le réel en devient quasi-magique, suspendu à ces écrits qui le font advenir comme par miracle.

Ce scénario écrit est comparable à un texte de théâtre qui se réduirait aux seules didascalies, souvent largement incomplètes. Parfois, rien ne dit qui fait l'action, parfois il n'y a qu'un prénom ou simplement la couleur d'un vêtement, les acteurs sont souvent interchangeables.

Le livret pointe ainsi les actions ayant valeur de « représentations » dans le flux indifférencié des multiples activités journalières. Il fabrique l'attente ludique de ces petits prodiges, telle une vision prémonitoire de ce qui va 
advenir. On déambule d'un lieu à l'autre, livret à la main. Et ce qui est écrit arrive, étrangement et sans coup férir. Drôle de prospective qui se réalise.

L'ensemble de l'intervention - livret de prévisions et actions - est une manière jouissive de fictionnaliser le réel et ses rituels en déplaçant notre point de vue sur le continuum des mille et une choses qui forment une journée. Étonnamment, l'événement se savoure malgré sa banalité : «Ah ! Voilà les deux cyclistes », «tiens, c'est ma voisine qui éteint la lumière de sa chambre $\gg$, etc. En rejouant le fragment, en extrayant et répétant le petit rien, la proposition œuvre par synecdoque : l'extrait pour la globalité, la partie pour le tout. Ce qui est écrit et donc joué vaut pour le réel car la fiction est si peu flagrante qu'elle en est presque invisible. Sauf à l'attendre et à la contempler.

L'achèvement du livret génère des applaudissements, les cris de joie des regardeurs, une satisfaction partagée. Le spectacle a donc bien eu lieu dans cette boucle où chacun a pu, à tour de rôle, être acteur et observateur.

Comment comprendre l'intérêt de cette intervention ? Une communauté qui se regarde vivre et fait de son quotidien la partition d'une représentation? Les objets, parce qu' ils sont rejoués, y prennent de l'intérêt et les participants, tels des enfants, entrent tour à tour dans le jeu ou s'en retirent pour regarder faire les autres. L'affaire est légère et les récalcitrants s'en tiennent à l'indifférence, ou à l'ignorance volontaire du scénario. Qui s'y refuse reste à l'écart mais ce faisant, continue à jouer de facto dans la pièce commune. La convention est si ténue qu'elle a du mal à créer son hors-champ.

Pourtant, à l'évidence, il y a re-présentation, endossement d'un rôle dans une logique de chacun son tour. L'univers du jeu est bien présent ; jeu du comédien qui s'écarte de sa nécessité d'être et endosse une figure - peu importe si la figure, ici, est la copie conforme du réel. Et jeu du gamin qui s'amuse, se divertit, invente la règle comme préalable plus ou moins implicite au « comme si... » collectif.

La définition que donne Roger Caillois du jeu ${ }^{7}$ est celle d'une activité libre, séparée, improductive, réglée et fictive, tous qualificatifs légitimes au regard de notre objet d'étude. Et il ajoute 《incertaine ». Là s'arrête l'analogie lorsque le jeu scénarisé relève du champ théâtral et installe dans le réel une pratique prévisible.

De manière générale, le jeu théâtral a profondément à voir avec la catégorie qu'il nomme mimicry, catégorie dont relève tout ce qui s'apparente au jeu de rôle, à l'endossement d'un personnage, à la création fictionnelle : pêle-mêle 
s'y retrouvent les enfants qui jouent au docteur, à la poupée, aux pirates, à la guerre des étoiles ou leurs aînés, fans des « grandeur nature » ou des reconstitutions historiques. La catégorie traverse les époques et les genres. Son principe consiste à fictionnaliser des objets, des lieux, des corps, des gestes à des fins de fabrication d'une narration plus ou moins cohérente mais $a$ minima admise par tous ceux qui la jouent et parfois par ceux qui la regardent.

On tient ici le concept qui ouvre vers la pratique théâtrale et signale une capacité à adhérer collectivement et sciemment à une fiction, à y participer, à s'en divertir.

Ici la fiction se glisse si bien dans le réel qu'elle pourrait être invisible sans cette conscience collective de refaire pour d'autres, puis d'être à son tour sur le banc pour regarder passer la vie, plus conscient peut-être de la ritualisation répétitive de ce microcosme où chacun est contraint par ses horaires de vie, de boulot, d'école, de voisinage. La redite organisée est une manière de donner à voir cet affairement entropique selon un principe fractal où l'échelle minuscule parle de l'ensemble. L'intervention de Grand Magasin est simplement le pas de côté, le décentrement qui donne ponctuellement à voir cette dimension itérative.

Toutefois, et selon les mots de Paul Valéry, « le jeu ne supporte pas le scepticisme $\gg$. Il ne fonctionne que si l'on accepte d'y entrer, d'entrer en croyance, in-lusio. L'action théâtrale à la fois fabrique les règles du jeu dans leur simplicité extrême mais ce faisant, dévoile peut-être l'ordre des choses et dit métaphoriquement d'autres règles plus souterraines qui fondent la réalité.

Règles celles-là invisibles sauf à se saisir d'autres outils critiques comme ceux de la sociologie d'Ervin Goffman que je voudrais convoquer ici.

Contrairement à la vision triviale qui considère que les individus agissent selon leur « nature », leur « personnalité » ou encore selon leur « humeur », Goffman appréhende l'ordre social comme un jeu d'interactions déterministes où les règles rendent possible le lien social. Et il s'appuie sur le jeu théâtral pour mieux faire comprendre ces interactions quotidiennes. La métaphore théâtrale a valeur ici d' « instrument heuristique qui permet de se distancier des relations ordinaires et d'en décrire les caractéristiques $\gg^{8}$.

Goffman, ce faisant, s'inscrit dans la tradition de Durkheim, où règles et rites apparaissent comme étant extérieurs aux consciences individuelles et exercent sur elles une contrainte. Si l'on adopte cette perspective, cela revient à dire que nous sommes pris dans un déterminisme qui emprunte au théâtre 
sa prédictibilité et l'assignation de chacun en ses rôles au sein d'échanges sociaux codifiés.

Goffman parle du jeu ${ }^{9}$ (social) au sens du théâtre, comme d'une pièce prévisible où les interactions seraient autant de règles qui nous privent de choix profonds, et non au sens des jeux enfantins certes liés par un scénario, mais incertains quant à leur devenir. Dans son monde, le jeu des interactions est déjà écrit.

Néanmoins, « ce qui rend possible la distance au rôle n'est donc pas l'existence d'un moi qui échapperait aux déterminations sociales, mais bien la multiplicité des rôles occupés par chaque individu $\gg^{10}$.

Dans ce type de théâtre ${ }^{11}$ où le réel (les gens, leurs actes, leurs espaces) reste la matière même qui est travaillée, la fabrique de nouveaux points de vue permet de se regarder vivre en rejouant ce réel. La matière se fait transfuge, se déplace et joue au sens où l'on dit d'une pièce de menuiserie qu'elle joue dans sa mécanique, qu'elle ne fonctionne pas - ou plus - comme on attendrait qu'elle le fasse parce que mal ajustée. Elle frotte, grince et se manifeste.

Goffman appelle cela $\ll$ la fausse note $\gg^{12}$ ou $\ll$ l'embarras $\gg^{13}$, non pas parce qu'il y met une connotation morale mais parce qu'il y voit une rupture $\mathrm{du}$ continuum déterministe. Ici et dans tout dispositif interventionniste du même type, nous repérons évidemment une force d'émancipation portée par cette mise en représentation. C'est la qualité même de Prévisions.

À ce jeu des règles qui déplacent le réel et le fictionnalisent, nous voudrions évoquer deux autres exemples, à la frontière des pratiques théâtrales et plasticiennes, où la pratique du rejeu nous semble signifiante et susceptible de venir enrichir l'analyse de notre premier exemple.

Sur son curriculum vitae, Nicolas Simarik écrit : « vit et travaille avec des gens $\gg$, là où d'autres indiquent leur lieu de résidence puis leur médium de prédilection. «Les gens » sont la matière revendiquée de son approche du réel. Certes, il émarge dans la catégorie des plasticiens mais la plupart de ses dispositifs relèvent de scénarios construits et performatifs, d'où sa présence légitime dans ce rapprochement.

En 2005, à l'initiative du collectif Entrez sans frapper, il s'installe pendant un an dans le quartier d'Empalot, à Toulouse, communément rangé dans les « zones urbaines sensibles ». L'objet produit lors de cette résidence est un pastiche du catalogue de vente par correspondance La Redoute ${ }^{14}$, nommé La Déroute. À première vue et formellement, la ressemblance est parfaite : 
deux kilos de papier, 1234 pages vendues au prix de l'original de l'année, soit $5,95 €$ pour un tirage à 15000 exemplaires. La maquette est visuellement semblable à l'original dans sa mise en page, sa typographie, la présence de slogans, la typologie des « rayons » singeant tous les poncifs; un vrai piège à regard. Les écarts pourtant très vite se révèlent en feuilletant l'objet : les gens sont des « vrais gens », ceux de cette cité. Pour cela, Nicolas Simarik s'est installé pendant un an dans ce quartier et a réalisé 22000 photos mettant en scène les 600 habitants du quartier, âgés de un mois à 104 ans, dans leurs appartements, sur les balcons, sur l'aire de jeux, autour d'une mobylette, d'une salle de gym, lors d'une sortie au ski etc., acteurs d'un catalogue qui ne vend rien sinon eux-mêmes. Les vieux sont vieux, les gros sont gros, on y voit des familles, des fratries, des copains. Les pages « maison » rentrent dans les appartements, dans les cuisines, dans l'intimité des chambres, présentent des meubles, des jouets. Les prix sont remplacés par l'heure de la prise de vue en conservant la typographie originelle et les pavés de textes égrènent en boucle les noms des participants. Le résultat est particulièrement jouissif : un magnifique portrait de groupe qui donne figure à ce lieu sans star, à cette communauté de vie habituellement sans visage. L'œuvre n'existe que par la volonté des habitants, l'artiste étant force de proposition mais dépourvu de la matière même de son travail sauf à susciter l'adhésion. Autour de cet objet, on imagine aisément l'immensité du travail préparatoire collectif, les rencontres humaines, l'affairement autour du studio provisoire installé entre les immeubles, les repas festifs, la convivialité, la chaleur informelle d'un lieu de vie où l'on se fait beau, le choix des tirages! Le projet donne des images à ceux qui n'en ont pas et ce faisant, rejoue différemment la réalité prise sous le regard de cette intervention.

Là encore, nous avons affaire à un objet artistique qui rejoue le réel sous forme de pastiche dans une dimension ouvertement ludique - activité libre, séparée, improductive, réglée et fictive ${ }^{15}$. La proposition de l'artiste vient réenchanter un objet commercial trivial et populaire dans une élaboration collective qui fictionnalise le réel. Il rédime l'existence quotidienne de ce groupe social en ouvrant de nouveaux rôles, inattendus et généreux, décalés et exceptionnels. Le pas de côté pour rejoindre l'analyse de Goffman.

« Alors on dirait que... »!

Plus radical, notre dernier exemple est un film, The Battle of Orgreave, réalisé en 2001 par un artiste anglais Jeremy Deller ${ }^{16}$; objet qui, là aussi, se présente 
sous une fausse apparence, celle de la reconstitution historique de bataille, genre bien connu des festivals estivaux et autres valorisations identitaires du patrimoine.

Orgreave est une commune du sud du Yorkshire, située à une douzaine de kilomètres de Sheffield. Le 18 juin 1984, Deller a 16 ans quand il assiste devant sa télévision au dernier affrontement entre les forces de police anglaises et les mineurs qui, depuis un an, défendent leur outil de travail contre celle que l'on appellera la Dame de fer, Margaret Thatcher, dans ce site ouvrier de charbonnerie et cokerie qu'est Orgreave. Lieu emblématique s'il en fut de ce conflit terrible qui eut raison des syndicats les plus puissants, divisa les familles et les amis dans une ambiance de guerre civile étendue à tout le pays, la bataille d'Orgreave a clôturé deux années de démantèlement d'un secteur économique dont on sait maintenant qu'il était productif et compétitif mais tenu par des syndicats très organisés ayant force de contre-pouvoir. À Orgreave, ce furent 15000 personnes qui se retrouvèrent face à la police $-\mathrm{y}$ compris face à la police montée - laissant sur le carreau de nombreux blessés graves et procédant à de multiples arrestations. Pour des raisons logistiques d'approvisionnement des autres lieux de production, Orgreave était un point de non-retour, symbole de cette dernière résistance sociale, cause définitivement perdue qui, malheureusement, laissa place à une quinzaine d'années de récession, de chômage et de crise.

Le travail de Jeremy Deller en 2001 oscille entre reconstitution historique et contre-documentaire. Pour les besoins du film, il fait rejouer à près de 800 personnes le scénario de cette lutte sans merci. La tradition anglaise du « re-enactement », ancrée de longue date dans les pratiques mémorielles entretenues par une certaine aristocratie, pourrait rendre la chose finalement assez banale et la renvoyer du côté des événements populaires. Mais la nature même du fait historique - un conflit social récent - n'en fait pas du tout un sujet éligible à ce type de manifestation. D’autre part, la plupart des figurants sont des vétérans du conflit, rejouant leur propre rôle ou celui de la police. À l'exception de quelques cascadeurs professionnels embauchés pour l'occasion, les acteurs sont ceux-là même qui ont vécu ces mois troubles, engagés eux-mêmes dans le conflit, ou via leur famille ou des gens très proches :

Pour beaucoup, qu'ils soient participants ou spectateurs, cette bataille d'Orgreave fut plus un flashback qu'une reconstitution. Sachant cela, les projectiles, les charges de la police montée, les coups, les défaites et les arrestations étaient bien plus que du spectacle; on oubliait facilement que les matraques de la police étaient en plastique, les pierres des mineurs en mousse et le sang coulant sur les visages, factice ${ }^{17}$. 
Ce qui s'y joue est loin d'être distancié : le film a beaucoup tourné ensuite et notamment dans toutes ces villes dévastées en 1984 par la fermeture des outils de production. Dix-sept ans plus tard, les plaies sont toujours vives et douloureuses, le film ouvrant toutefois un espace de dialogue et de catharsis au sein de communautés mal réconciliées ou auprès des jeunes générations. En 1984, les médias eux-mêmes étaient assujettis à l'emprise gouvernementale, occultant la parole de cette vague de fond populaire, écartée des antennes. Le film de Deller rend également des images et des mots à ceux qui en avaient manqué.

Actuellement, l'objet se présente sous la forme d'un DVD et d'un livre, compilant le film et les documents, les interviews, les témoignages. Deller revendique cette forme de «living history $\gg^{18}$ telle qu'elle se pratique beaucoup en Angleterre et la présence de cascadeurs professionnels était pour lui un gage de cette légitimité recherchée :

Je voulais embaucher des membres de ces sociétés essentiellement pour deux raisons : la première c'est qu'ils étaient entrainés à la reconstitution de combats et à l'obéissance aux ordres. Mais surtout, je voulais que la reconstitution de La Bataille d'Orgreave s'inscrive dans la lignée des batailles décisives de l'histoire de l'Angleterre ${ }^{19}$.

Et plus loin :

Je ne me serais jamais lancé dans ce projet si la population locale l'avait trouvé inutile ou de mauvais goût. Tel qu'il était proposé, nous avons été soutenus dès le début parce qu'il semblait y avoir une compréhension instinctive de ce qui allait se jouer dans cette reconstitution. Je n'étais pas intéressé par une interprétation nostalgique de la grève ${ }^{20}$.

Scénariser, mettre en scène et filmer la représentation de cette journée emblématique avec des acteurs/auteurs de l'histoire originale donne à cet objet une valeur paradigmatique en ce qu'il décentre une mémoire blessée, marquée par des déchirements fratricides : rejouer mais en changeant de place, en changeant de rôle pour mieux appréhender la complexité d'une histoire confuse et chaotique, pour mieux la distancier et en tenir les enjeux. Les plaies restent profondes, là où le conflit a pris des allures de guerre civile. $\mathrm{Si}$ les années ont passé, reprendre sa place a valeur cathartique, ouvre la parole là où elle n'a pu advenir. Des mineurs deviennent policiers et inversement pour les besoins du tournage, les rangs bougent, des enfants prennent la place des parents, des professionnels soutiennent la bagarre. Le rejeu nécessite une distance scénographique où l'expérience primitive est racontée, partagée mais 
aussi déchargée de sa violence, laissant à la caméra le soin de tenir la fiction et de faire l'histoire.

De ces trois objets - légers, drôles ou graves -, nous pouvons dire qu'ils mettent en œuvre des dispositifs de jeux de rôles à valeur autoréflexive. Jouer le réel / jouer avec le réel est une manière de se l'approprier, d'en faire une forme d'épistémologie en acte. Tel est le décentrement proposé par les artistes, qui éclaire le territoire de l'expérience, loin d'une télé réalité qui se contente d'un regard voyeur et ne donne en rien les outils d'une lecture critique. Ce décentrement ici repéré provoque une « rupture de cadres » à savoir une défaillance des indicateurs ordinaires, où la nature même de nos croyances se trouve bouleversée, éclairant ainsi par la négative le rôle rempli par les cadres dans les interactions quotidiennes.

Jouer, ici, c'est ouvrir la pensée sur le réel si proche, c'est donner à voir des expériences paradigmatiques qui éclairent le continuum indifférencié, la confusion ordinaire.

C'est pour cela que jouent les enfants, pour fabriquer à leur mesure le rejeu fictionnel d'une existence dont ils s'arrogent ainsi provisoirement la maîtrise.

C'est pour cela que quelques artistes poursuivent l'infiltration du réel et produisent des objets de l'entre-deux, fabriquent des pseudo-mondes dont les règles, absurdes ou graves, ne parlent de rien d'autre que de nos vies, mais contemplées à neuf, avec la distance critique du jeu théâtral qui libère les rôles.

\section{Notes}

1. [En ligne], Grand Magasin [page consultée le $1^{\text {er }}$ juin 2016], disponibilité et accès http://www.grandmagasin.net/spectacle.php?spec=22.

2. Ibid.

3. Ibid.

4. Extraits du livret accompagnant l'intervention. La mise en ligne ainsi que les majuscules ont été conservées.

5. Enrôler des éléments que l'on ne maîtrise pas et que l'on s'approprie alors qu'ils sont totalement hors de portée est une pratique confiscatoire savoureuse déjà utilisée par 
Rejouer la ville, rejouer le réel : Grand Magasin ou les enjeux de la prévision

d'autres. On pense à Gianni Motti revendiquant comme relevant de son œuvre des tremblements de terre (Californie, juin 1992) ou l'explosion de la navette Challenger (1986).

6. On lira une belle analyse de ce phénomène de quasi-invisibilité urbaine dans l'ouvrage de Marie Escorne, L'Art à même la ville (Pessac, Presses Universitaires de Bordeaux, 2015, p. 47-49) à propos des Actions-peu de l'artiste plasticien Boris Achour.

7. R. Caillois, Les Jeux et les hommes, le masque et le vertige, Paris, Gallimard, coll. « Folio Essais », 1967 [1958]. Le chapitre 1 est consacré à cette définition. Nous en résumons les principes.

8. M. Thura, «Une réévaluation de la métaphore théâtrale chez Erving Goffman », Revue de synthèse, t. 133, $\mathrm{n}^{\circ}$ 4, 2012, p. 577.

9. Deux ouvrages de Goffman se réfèrent de manière centrale à la métaphore du jeu : Encounters (1961) et Strategic Interactions (1969).

10. J. Nizet et N. Rigaux, La Sociologie de Erving Goffman, Paris, La Découverte, coll. " Repères ", 2014, p. 23. Je souligne.

11. Tel que le pratique Prévisions.

12. Cf. Erving Goffman, La Mise en scène de la vie quotidienne. 1. La Présentation de soi, Paris, Minuit, coll. «Le Sens commun », 1973, p. 229.

13. Cf. Erving Goffman, Les Rites d'interaction, Paris, Minuit, coll. « Le Sens commun », 1998, p. 87-101.

14. Pas de hasard dans le choix de ce pastiche. Les catalogues de vente par correspondance ont été pour toute une génération de formidables réserves d'images, panoplies d'un monde idéal où, enfants, nous découpions et recomposions nos propres fictions, matière première de jeux pas chers et de rêves à portée de ciseaux.

15. Nous reprenons les caractéristiques extraites de l'ouvrage de Roger Caillois.

16. Jeremy Deller, The Battle of Orgreave, réalisé par Mike Figgis, The Artangel collection, 2001. Le film peut être visionné sur Viméo ou sur YouTube.

17. "For many - participants and spectators alike - this Battle of Orgreave was more flashback than re-enactment. Knowing this made the missiles, the mounted police charges, the beatings, routs and arrests much more than spectacle; it was easy to forget the police's truncheons were plastic, the miners' rocks just foam, and that the blood running down some faces was fake ". Alex Farquharson, publié dans Frieze, septembre 2001. Je traduis.

18. Le concept de « living history » témoigne de l'attrait des Anglo-saxons pour les reconstitutions de batailles et d'épisodes remarquables. De la conquête romaine à la Seconde Guerre mondiale en passant par la bataille d'Hastings, ce sont des événements qui attirent les foules et génèrent un marché très spécifique : metteurs en scène et cascadeurs spécialisés, productions ou reproductions d'armes, de costumes, d'accessoires etc. Cette richesse événementielle recouvre souvent des enjeux politiques souverainistes ou régionalistes et exalte la fibre patriotique.

19. "I wanted to involve members of these societies for mainly two reasons: first of all, they are well trained in recreating combat and in obeying orders. More importantly, I wanted the re-enactment of The Battle of Orgreave to become part of the lineage of decisive battles in English History. ». Artangel [En ligne], Artangel éditeur, [Page consultée le 2 juin 2016]. Disponibilité et accès : https://www.artangel.org.uk/project/the-battle-of-orgreave/.Je traduis. 
Théâtres du geste, du jeu et de la voix

20. "I would never have undertaken the project if people locally felt it was unnecessary or in poor taste, As it was, we encountered support from the outset because there seemed to be an instinctive understanding of what the re-enactment was about. I was not interested in a nostalgic interpretation of the strike ». Artangel [En ligne], Artangel éditeur, [Page consultée le 2 juin 2016]. Disponibilité et accès : https://www.artangel.org.uk/project/the-battle-of-orgreave/. Je traduis. 